\title{
Electromagnetic properties of strange baryons in a relativistic quark model
}

\author{
T. Van Cauteren ${ }^{1, a}$, J. Ryckebusch ${ }^{1}$, B. Metsch ${ }^{2}$, and H.-R. Petry ${ }^{2}$ \\ 1 Department of Subatomic and Radiation Physics, Ghent University, Proeftuinstaat 86, B-9000 Gent, Belgium \\ 2 HISKP, Bonn University, Nußallee 14-16, D-53115 Bonn, Germany
}

Received: 23 November 2006

Published online: 16 March 2007 - (C) Società Italiana di Fisica / Springer-Verlag 2007

\begin{abstract}
We present some of our results for the electromagnetic properties of excited $\Sigma$ hyperons, computed within the framework of the Bonn constituent-quark model, which is based on the Bethe-Salpeter approach. The seven parameters entering the model are fitted against the best-known baryon masses. Accordingly, the results for the form factors and helicity amplitudes are genuine predictions. We compare with the scarce experimental data available and discuss the processes in which $\Sigma^{*}$ 's may play an important role.
\end{abstract}

PACS. 11.10.St Bound and unstable states; Bethe-Salpeter equations - 12.39.Ki Relativistic quark model - 13.40.Gp Electromagnetic form factors - 14.20.Jn Hyperons

\section{Introduction}

Strangeness physics is gaining more and more momentum in present-day hadronic physics. Due to the upgrading of existing and the commisioning of new ones, more experimental facilities than ever can perform measurements of hyperon structure and interactions.

Reactions such as the electromagnetic production of kaons or radiative kaon capture are sensitive to the electromagnetic (EM) properties of the particles involved. While for the low-lying nonstrange hadrons, a reasonable amount of data is available, the EM properties of hyperons and hyperon resonances are almost completely unknown. Yet modelling the above-mentioned reactions requires knowledge of the strength with which $Y^{(*)}$ 's couple to photons. The work presented here and in refs. [1,2] tries to remedy this gap of knowledge by computing the EM form factors of $Y^{(*)}$ 's in the framework of the covariant quark model developed by the Bonn group [3]. This constituent-quark (CQ) model is based on the Bethe-Salpeter approach and uses instantaneous interactions: confinement is ensured by a linearly rising $\Delta$-shaped potential and hyperfine splittings are accounted for by an instanton-induced interaction. It should be mentioned that lattice-QCD calculations show a preference for a $Y$-shaped confinement potential between three static quarks at distances larger than $\sim 0.8 \mathrm{fm}[4]$. However, the effects of assuming a different confinement ansatz (and subsequently rescaling the confinement parameters) seem to be rather small and at this

\footnotetext{
a e-mail: Tim.VanCauteren@UGent.be
}

moment beyond experimental verification, both for the spectrum as for EM form factors. The seven parameters in the model are fitted to the masses of the best-known baryon resonances, but no extra parameters are used in computing the EM form factors. The calculations are performed consistently at first order to obtain the vertexfunctions $\Gamma\left(p_{\xi}, p_{\eta}\right)$ as amputated Bethe-Salpeter amplitudes describing the baryon states in terms of the Jacobi momenta $p_{\xi}$ and $p_{\eta}$. The EM current matrix elements are then computed in lowest order in the rest frame of the incoming state according to $[1,2,5]$

$$
\begin{aligned}
& \left\langle\bar{P}\left|j^{\mu}(0)\right| M\right\rangle \simeq(-3) \int \frac{\mathrm{d}^{4} p_{\xi}}{(2 \pi)^{4}} \frac{\mathrm{d}^{4} p_{\eta}}{(2 \pi)^{4}} \bar{\Gamma} \frac{\Lambda}{P}\left(p_{\xi}, p_{\eta}\right) \\
& \quad \times S_{F}^{1}\left(\frac{M}{3}+p_{\xi}+\frac{p_{\eta}}{2}\right) \otimes S_{F}^{2}\left(\frac{M}{3}-p_{\xi}+\frac{p_{\eta}}{2}\right) \\
& \otimes\left[S_{F}^{3}\left(\frac{M}{3}-p_{\eta}+q\right) \hat{q} \gamma^{\mu} S_{F}^{3}\left(\frac{M}{3}-p_{\eta}\right)\right] \\
& \quad \times \Gamma_{M}^{\Lambda}\left(p_{\xi}, p_{\eta}+\frac{2}{3} q\right),
\end{aligned}
$$

where $\bar{P}$ is the total four-momentum of the outgoing onshell state, $M$ is the mass of the incoming state, $S_{F}^{i}(p)$ denotes the propagator for the $i$-th CQ, and $\hat{q}$ is the CQcharge operator. The operator $\otimes$ denotes a direct product in the $(q q q)$-space. From this matrix element, the EM form factors or helicity amplitudes (HAs) can be derived. The limits of the current matrix elements for $Q^{2} \rightarrow 0$ give the 
response of the baryon to real photons and are needed to compute EM decay widths.

\section{Helicity amplitudes}

The helicity amplitudes presented here are defined by [2]

$$
\begin{gathered}
A_{1 / 2}\left(\Sigma^{*} \rightarrow Y\right)= \\
\mathcal{D}\left\langle Y, \bar{P}, \frac{1}{2}\left|j^{1}(0)+i j^{2}(0)\right| \Sigma^{*}, \bar{P}^{*},-\frac{1}{2}\right\rangle, \\
A_{3 / 2}\left(\Sigma^{*} \rightarrow Y\right)= \\
\mathcal{D}\left\langle Y, \bar{P},-\frac{1}{2}\left|j^{1}(0)+i j^{2}(0)\right| \Sigma^{*}, \bar{P}^{*},-\frac{3}{2}\right\rangle, \\
C_{1 / 2}\left(\Sigma^{*} \rightarrow Y\right)=\mathcal{D}\left\langle Y, \bar{P}, \frac{1}{2}\left|j^{0}(0)\right| \Sigma^{*}, \bar{P}^{*}, \frac{1}{2}\right\rangle,
\end{gathered}
$$

for the EM transitions between excited $\left(\Sigma^{*}\right)$ and groundstate $(Y)$ hyperons. Here, $\mathcal{D}=\sqrt{\frac{\pi \alpha}{2 m\left(m^{* 2}-m^{2}\right)}}$ depends on the specific normalization used for the vertex functions. The EM decay width for the process $\Sigma^{*} \rightarrow Y+\gamma$ is then given by

$$
\Gamma_{\gamma}=\frac{|\mathbf{q}|^{2}}{4 \pi^{2} \alpha} \frac{2 m}{\left(2 J^{*}+1\right) m^{*}}\left[\left|A_{1 / 2}\right|^{2}+\left|A_{3 / 2}\right|^{2}\right],
$$

where $|\mathbf{q}|=\frac{m^{* 2}-m^{2}}{2 m^{*}}$ is the photon three-momentum in the rest frame of the initial baryon resonance, and $\alpha=\frac{e^{2}}{4 \pi} \simeq$ $\frac{1}{137}$ is the EM fine-structure constant. Remark that this definition differs from the PDG [6] by a factor of $e^{2}=4 \pi \alpha$. Wherever we can, we will compare our calculations with experiment. However, since we only compute EM current matrix elements, and not e.g. pion decay, only the relative signs between the three HAs of a certain $\Sigma^{*} \rightarrow Y$ decay are fixed. If the ground-state hyperon is a $\Sigma$, the relative HAs for the different isospin channels are also determined.

\section{Results for the $J=3 / 2 \Sigma^{*}$-resonances}

Due to limited space, we only show the HAs of the $\Sigma^{*-}+\gamma^{*} \rightarrow \Sigma^{-}$decays for lowest-lying spin $J=3 / 2$ resonances in fig. 1. A few observations can be made here. First, in contrast to most elastic EM form factors [1], some HAs are larger at non-zero $Q^{2}$ than at the real-photon point. This property is more general than in the specific decays shown here and also turns up in other HAs both in the nonstrange [5] and in the hyperon sector [2]. It is therefore necessary to include electroproduction processes in the search for baryon resonances, and not only photoproduction reactions.

Another observation one can infer from fig. 1 is that there are no HAs with a magnitude larger than $100 \times$ $10^{-3} \mathrm{GeV}^{-1 / 2}$. Therefore, it is better to look for signals of spin $J=3 / 2 \Sigma$-resonances in the positively charged isospin channel than in the negatively charged one. This
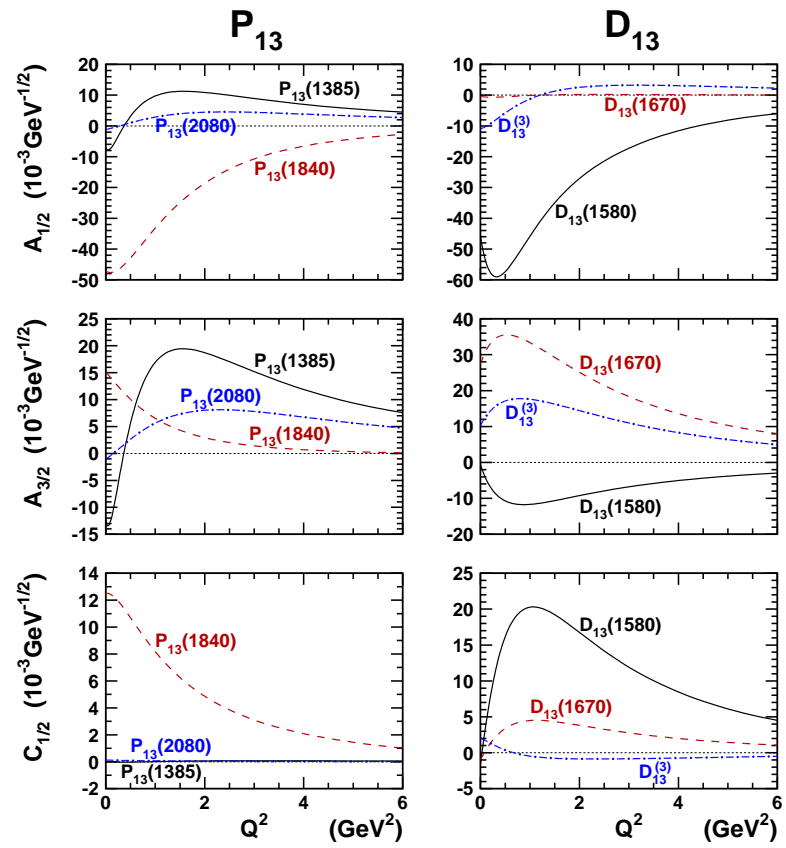

Fig. 1. The $Q^{2}$-dependence for the $\Sigma^{*-}+\gamma^{*} \rightarrow \Sigma^{-}$decays for spin $J=3 / 2$ resonances: left (right) panels show the results for the positive (negative) parity $\Sigma^{*}$-resonances.

Table 1. Calculated masses, photo-amplitudes and EM decay widths for the $\Sigma^{*}+\gamma \rightarrow \Sigma(1193)$ transitions for $J=3 / 2$ $\Sigma^{*}$-resonances. The charge of the $\Sigma^{*}$ isospin-triplet member is indicated by the superscripts $0,+,-$. Masses and decay widths are given in units of $\mathrm{MeV}$, photo-amplitudes are given in units of $10^{-3} \mathrm{GeV}^{-1 / 2}$. The value in parentheses is the experimental upper limit quoted by the PDG [6], with a $90 \%$ confidence level.

\begin{tabular}{|c|c|c|c|c|}
\hline Resonance & $M_{\text {calc }}$ & $A_{1 / 2}$ & $A_{3 / 2}$ & $\Gamma_{\text {calc }}$ \\
\hline \hline$P_{13}^{0}(1385)$ & 1409 & 27.8 & 48.0 & 0.181 \\
$P_{13}^{0}(1840)$ & 1902 & 15.4 & -5.25 & 0.0960 \\
$P_{13}^{0}(2080)$ & 1950 & 14.3 & 23.7 & 0.303 \\
$D_{13}^{0}(1580)$ & 1675 & -2.82 & -32.9 & 0.230 \\
$D_{13}^{0}(1670)$ & 1727 & -6.77 & -6.45 & 0.0214 \\
$D_{13}^{(3) 0}$ & 1780 & 24.4 & 25.5 & 0.349 \\
\hline$P_{13}^{+}(1385)$ & 1409 & 62.6 & 108.2 & 0.920 \\
$P_{13}^{+}(1840)$ & 1902 & 80.0 & -25.6 & 2.559 \\
$P_{13}^{+}(2080)$ & 1950 & 29.7 & 48.5 & 1.280 \\
$D_{13}^{+}(1580)$ & 1675 & 40.0 & -65.2 & 1.235 \\
$D_{13}^{+}(1670)$ & 1727 & -13.1 & -40.3 & 0.440 \\
$D_{13}^{(3)+}$ & 1780 & 59.8 & 40.8 & 1.468 \\
\hline$P_{13}^{-}(1385)$ & 1409 & -7.06 & -12.2 & 0.0117 \\
& & & & $(<0.0095 \pm 0.0006)$ \\
$P_{13}^{-}(1840)$ & 1902 & -47.1 & 15.1 & 0.887 \\
$P_{13}^{-}(2080)$ & 1950 & -1.20 & -1.05 & 0.00101 \\
$D_{13}^{-}(1580)$ & 1675 & -45.7 & -0.588 & 0.441 \\
$D_{13}^{-}(1670)$ & 1727 & -0.397 & 27.4 & 0.184 \\
$D_{13}^{(3)-}$ & 1780 & -10.9 & 10.3 & 0.0630 \\
\hline
\end{tabular}


is better illustrated in table 1 where the computed decay widths are shown for all the isospin channels in the $\Sigma^{*}(J=3 / 2) \rightarrow \Sigma$ EM decay. The predicted EM decays of the positively charged resonances are clearly larger than for the other channels.

Unfortunately, the experimental data on EM properties of $\Sigma$-resonances is very scarce. In fact, the only data mentioned in the PDG tables [6] are the EM decay width of the $\Sigma^{* 0}(1385) \rightarrow \Lambda(1116)\left(479 \pm 120_{-100}^{+81} \mathrm{keV}\right)$ and an upper limit for the EM decay width of the $\Sigma^{*-}(1385) \rightarrow \Sigma^{-}(1193)$ process $(<9.5 \pm 0.6 \mathrm{keV}$ at $90 \%$ confidence level). The former was extracted from looking at $p \gamma \rightarrow K^{+} \Lambda \gamma$ processes [7] at Jefferson Lab. Therefore, one may hope to extract information on the $\Sigma^{*+}(1385) \rightarrow$ $\Sigma^{+}$(1193) EM decay width from $p \gamma \rightarrow K^{0} \Sigma^{+} \gamma$ reactions. Our computed value of the $\Sigma^{* 0} \rightarrow \Lambda$ EM decay width is $1.53 \mathrm{MeV}$, which is a factor of three larger than the measured value. In table 1 , one can see that also our value for the $\Sigma^{*-} \rightarrow \Sigma^{-}$EM decay width is slightly larger than the upper limit given in the PDG. This overestimation of the EM decay widths could be due to meson loop or exchange current contributions to the process not included in the present model.

\section{Isospin asymmetries for the $\mathrm{J}=3 / 2$ $\Sigma^{* 0}$-resonances}

The isospin asymmetries are defined as

$$
\begin{gathered}
\mathcal{T}_{1 / 2}=\frac{\left|A_{1 / 2}^{\Lambda}\right|^{2}-\left|A_{1 / 2}^{\Sigma}\right|^{2}}{\left|A_{1 / 2}^{\Lambda}\right|^{2}+\left|A_{1 / 2}^{\Sigma}\right|^{2}}, \\
\mathcal{T}_{3 / 2}=\frac{\left|A_{3 / 2}^{\Lambda}\right|^{2}-\left|A_{3 / 2}^{\Sigma}\right|^{2}}{\left|A_{3 / 2}^{\Lambda}\right|^{2}+\left|A_{3 / 2}^{\Sigma}\right|^{2}}, \\
\mathcal{T}_{0}=\frac{\left|C_{1 / 2}^{\Lambda}\right|^{2}-\left|C_{1 / 2}^{\Sigma}\right|^{2}}{\left|C_{1 / 2}^{\Lambda}\right|^{2}+\left|C_{1 / 2}^{\Sigma}\right|^{2}} .
\end{gathered}
$$

Here, the superscript $\Lambda(\Sigma)$ stands for the decay of the resonance to the $\Lambda\left(\Sigma^{0}\right)$ ground state. It is clear that a positive (negative) value indicates that the resonance will preferentially decay to the $\Lambda\left(\Sigma^{0}\right)$ ground state.

In fig. 2 the computed isospin asymmetries are displayed for the lowest-lying $J=3 / 2 \Sigma^{* 0}$-resonances. With the exception of the $P_{13}(1840)$ and the $D_{13}(1670)$, the shown asymmetries tend to drop with increasing $Q^{2}$. Therefore the $\Sigma^{* 0}$-resonances will couple mostly to the $\gamma^{*} \Lambda$ channel at low $Q^{2}$, and to the $\gamma^{*} \Sigma^{0}$ channel at intermediate to high $Q^{2}$. The $\mathcal{T}_{1 / 2^{-}}$and $\mathcal{T}_{3 / 2}$-asymmetries of the $P_{13}(1840)$ behave in the opposite way. The $D_{13}(1670)$ decays preferentially to the $\gamma^{(*)} \Lambda$ channel at vanishing and small $Q^{2}$. The mass of this resonance is in the kinematic region of the $K^{-} p \rightarrow \gamma Y^{0}$ experiments investigated by
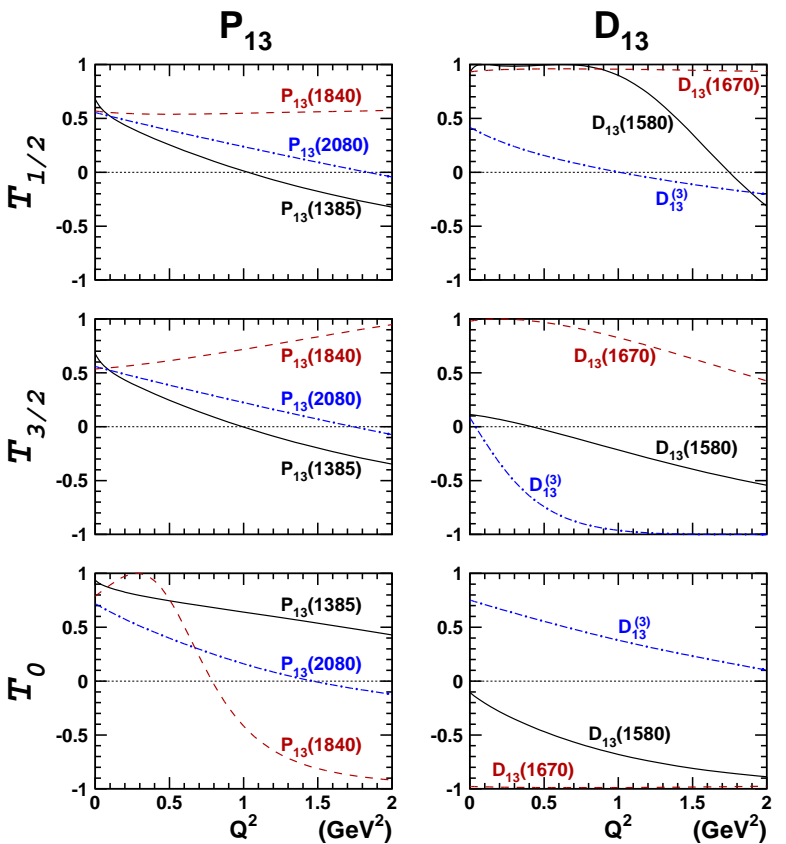

Fig. 2. The isospin asymmetries for $0<Q^{2}<2.0 \mathrm{GeV}^{2}$ as defined in eqs. (4) for the EM decays to different isospin channels for the three lowest-lying $(J=3 / 2, S=-1, I=1)$ $\Sigma^{* 0}$-resonances with positive-parity (left panels) and negativeparity (right panels).

the Crystal Ball Collaboration [8]. From our calculations one would expect that this resonance may only be seen in the $\gamma \Lambda$ channel and not in the $\gamma \Sigma^{0}$ channel.

\section{References}

1. T. Van Cauteren, D. Merten, J. Ryckebusch, T. Corthals, S. Janssen, B. Metsch, H.-R. Petry, Eur. Phys. J. A 20, 283 (2004).

2. T. Van Cauteren, J. Ryckebusch, B. Metsch, H.-R. Petry, Eur. Phys. J. A 26, 339 (2005).

3. U. Löring, K. Kretzschmar, B. Metsch, H.-R. Petry, Eur. Phys. J. A 10, 309 (2001); U. Löring, B. Metsch, H.-R. Petry, Eur. Phys. J. A 10, 395; 447 (2001).

4. Ph. de Forcrand, O. Jahn, Nucl. Phys. A 755, 475c (2005).

5. D. Merten, U. Löring, K. Kretzschmar, B. Metsch, H.-R. Petry, Eur. Phys. J. A 14, 477 (2002).

6. W.-M. Yao et al., J. Phys. G 33, 1 (2006).

7. S. Taylor et al., Phys. Rev. C 71, 054609 (2005); 72, $039902(2005)(\mathrm{E})$.

8. S. Prakhov, Progress on Study of $K^{-}$-Proton Reactions (UCLA, USA, 2001) http://bmkn8.physics.ucla.edu/ Crystalball/Docs/documentation.html, unpublished; N. Phaisangittisakul, First Measurement of the Radiative Process $K^{-} p \rightarrow \Lambda \gamma$ at Beam Momenta $520-750 \mathrm{MeV} / c$ Using the Crystal Ball Detector, PhD Thesis, University of California, Los Angeles, USA (2001). 